\title{
Influence of magnification threshold on pixel lensing optical depth, event rate and time scale distributions towards M 31
}

\author{
F. De Paolis ${ }^{1}$, G. Ingrosso ${ }^{1}$, A. A. Nucita ${ }^{1}$, and A. F. Zakharov ${ }^{2,3}$ \\ ${ }^{1}$ Dipartimento di Fisica, Università di Lecce and INFN, Sezione di Lecce, CP 193, 73100 Lecce, Italy \\ e-mail: ingrosso@le.infn.it \\ 2 Institute of Theoretical and Experimental Physics, 25, B. Cheremushkinskaya St., Moscow 117259, Russia \\ 3 Astro Space Centre of Lebedev Physics Institute, Moscow
}

Received 26 May 2004 / Accepted 2 November 2004

\begin{abstract}
Pixel lensing is the gravitational microlensing of light from unresolved stars contributing to the luminosity flux collected by a single pixel. A star must be sufficiently magnified, that is, the lens impact parameter must be less than a threshold value $u_{\mathrm{T}}$ if the excess photon flux in a pixel is to be detected over the background. Assuming the parameters of the Isaac Newton Telescope and typical observing conditions, we present maps in the sky plane towards M31 of threshold impact parameter, optical depth, event number and event time scale, analyzing in particular how these quantities depend on $u_{\mathrm{T}}$ in pixel lensing searches. We use an analytical approach consisting of averaging on $u_{\mathrm{T}}$ and the star column density the optical depth, microlensing rate and event duration time scale. An overall decrease in the expected optical depth and event number with respect to the classical microlensing results is found, particularly towards the high luminosity M 31 inner regions. As expected, pixel lensing events towards the inner region of M31 are mostly due to self-lensing, while in the outer region dark events dominate even for a $20 \%$ MACHO halo fraction. We also find a far-disk/near-disk asymmetry in the expected event number, smaller than that found by Kerins (2004). Both for self and dark lensing events, the pixel lensing time scale we obtain is $\simeq 1-7$ days, dark events lasting roughly twice as long as self-lensing events. The shortest events are found to occur towards the M 31 South Semisphere. We also note that the pixel lensing results depend on $\left\langle u_{\mathrm{T}}\right\rangle$ and $\left\langle u_{\mathrm{T}}^{2}\right\rangle$ values and ultimately on the observing conditions and telescope capabilities.
\end{abstract}

Key words. gravitational lensing - Galaxy: halo - cosmology: dark matter - galaxies: individual: M31 - methods: observational

\section{Introduction}

Pixel lensing surveys towards M 31 (Crotts 1992; Baillon et al. 1993) can give valuable information to probe the nature of MACHOs (Massive Astrophysical Compact Halo Objects) discovered in microlensing experiments towards the LMC and SMC (Large and Small Magellanic Clouds) (Alcock et al. 1993; Aubourg et al. 1993) and also address the question of the fraction of halo dark matter in the form of MACHOs in spiral galaxies (Alcock et al. 2000).

This may be possible due to both the increase in the number of expected events and because the M 31 disk is highly inclined with respect to the line of sight and so microlensing by MACHOs distributed in a roughly spherical M 31 halo give rise to an unambiguous signature: an excess of events on the far side of the M31 disk relative to the near side (Crotts 1992).

Moreover, M 31 surveys probe the MACHO distribution in a different direction to the LMC and SMC and observations are made from the North Earth hemisphere, probing the entire halo extension.
The Pixel lensing technique studies the gravitational microlensing of unresolved stars (Ansari et al. 1997). In a dense field of stars, many of them contribute to each pixel. However, if one unresolved star is sufficiently magnified, the increase of the total flux will be large enough to be detected. Therefore, instead of monitoring individual stars as in classical microlensing, one follows the luminosity intensity of each pixel in the image. When a significative (above the background and the pixel noise) photon number excess repeatedly occurs, it is attributed to an ongoing microlensing event if the pixel luminosity curve follows (as a function of time) a Paczynski like curve (Paczynski 1996).

Clearly, variable stars could mimic a microlensing curve. These events can be recognized by performing observations in several spectral bands and monitoring the signal from the same pixel for several observing seasons to identify the source.

Two collaborations, MEGA (preceded by the VATT/Columbia survey) and AGAPE have produced a number of microlensing event candidates, which show a rise in pixel luminosity in M31 (Crotts \& Tomaney 1996; Ansari et al. 1999; Auriere et al. 2001; Calchi Novati et al. 2002). 
Table 1. Parameters for the four M 31 models considered by Kerins (2004). Columns are the model name, the component name, the mass of the component, its central density $\rho_{0}$ and the adopted cut-off radius $R$. Additional columns give, where appropriate, the core radius $a$, the disk scale length $h$ and height $H$, the flattening parameter $q$ and the $B$-band mass-to-light ratio $M / L_{\mathrm{B}}$ in solar units.

\begin{tabular}{cccccccccc}
\hline \hline Model & Component & $\begin{array}{c}\text { Mass } \\
\left(\times 10^{10} M_{\odot}\right)\end{array}$ & $\begin{array}{c}\rho_{0} \\
\left(\times M_{\odot} \mathrm{pc}^{-3}\right)\end{array}$ & $\begin{array}{c}R \\
(\mathrm{kpc})\end{array}$ & $\begin{array}{c}a \\
(\mathrm{kpc})\end{array}$ & $\begin{array}{c}h \\
(\mathrm{kpc})\end{array}$ & $\begin{array}{c}H \\
(\mathrm{kpc})\end{array}$ & $q$ & $M / L_{\mathrm{B}}$ \\
\hline Massive halo & halo & 191 & 0.25 & 155 & 2 & - & - & 1 & - \\
& bulge & 4.4 & 4.5 & 40 & 1 & - & - & 0.6 & 9 \\
& disk & 3.2 & 0.24 & 40 & - & 6 & 0.3 & - & 4.5 \\
\hline Massive bulge & halo & 89 & 0.01 & 85 & 10 & - & - & 1 & - \\
& bulge & 8 & 2.5 & 40 & 1.5 & - & - & 0.6 & 14 \\
& disk & 11 & 0.6 & 40 & - & 7 & 0.3 & - & 18 \\
\hline Massive disk & halo & 79 & 0.01 & 110 & 8 & - & - & 1 & - \\
& bulge & 4.4 & 4.5 & 40 & 1 & - & - & 0.6 & 9 \\
& disk & 19 & 1.4 & 40 & - & 6 & 0.3 & - & 26 \\
\hline Reference & halo & 123 & 0.065 & 100 & 4 & - & - & 1 & - \\
& bulge & 4.4 & 4.5 & 40 & 1 & - & - & 0.6 & 9 \\
& disk & 5.3 & 0.35 & 40 & - & 6.4 & 0.3 & - & 8 \\
\hline
\end{tabular}

More recently, based on observations with the Isaac Newton Telescope on La Palma (Kerins et al. 2001), the MEGA (de Jong et al. 2004), POINT-AGAPE (Paulin-Henriksson et al. 2003; Calchi Novati et al. 2003; Uglesich et al. 2004) and WeCAPP (Riffeser et al. 2003) collaborations claimed to find evidence of several microlensing events.

In particular, the MEGA Collaboration (de Jong et al. 2004) presented the first $14 \mathrm{M} 31$ candidate microlensing events, 12 of which are new and 2 that have been reported by the POINTAGAPE Collaboration (Paulin-Henriksson et al. 2003). The preliminary analysis of the spatial and timescale distribution of the events supports their microlensing nature. In particular the far-disk/near-disk asymmetry, although not highly significant, is suggestive of the presence of an M 31 dark halo.

The POINT-AGAPE Collaboration found in total a subset of four short timescale, high signal-to-noise ratio microlensing candidates, one of which is almost certainly due to a stellar lens in the bulge of M 31 and the other three candidates can be explained either by stars in M31 and M32, or by MACHOs.

In pixel lensing surveys, although all stars contributing to the same pixel are candidates for a microlensing event, only the brightest stars (usually blue and red giants) will be magnified enough to be detectable above background fluctuations (unless for very high amplification of main sequence stars, which are very unlikely events).

First evaluations have shown that the pixel lensing technique towards M 31 may give rise to a significant number of events due to the large number of stars contributing to the same pixel (Baillon et al. 1993; Jetzer 1994; Gould 1994; Colley 1995; Han \& Gould 1996).

Although these analytic estimates may be very rough, they provide useful qualitative insights. To have reliable estimates in true observational conditions one should use Monte-Carlo simulations (Ansari et al. 1997; Kerins et al. 2001). In this way, given the capabilities of the telescope and CCD camera used, the observing campaign and weather conditions, one can estimate the event detection efficiency as a function of event duration and maximum amplification.
This study will be done in a forthcoming paper (De Paolis et al. 2004) with the aim of investigating the lens nature (i.e. the population to which the lens belongs) for the events discovered by MEGA (de Jong et al. 2004) and POINT-AGAPE (Paulin-Henriksson et al. 2003).

In this paper, instead of using Monte-Carlo simulations, we estimate the relevant pixel lensing quantities by analyzing the effect of the presence of a magnification threshold (or, equivalently, of a threshold impact parameter $u_{\mathrm{T}}$ ) in pixel lensing searches towards M31. We use an analytic procedure consisting of averaging the classical optical depth, microlensing rate and event duration time scale on $u_{\mathrm{T}}$, which depends on the magnitude of the source being magnified.

The paper is organized as follows. In Sect. 2 we briefly discuss the source - bulge and disk stars in M 31 - and lens - stars in M 31 and in the Milky Way (MW) disk, MACHOs in M 31 and MW halos - models we use. In Sect. 3 we discuss the pixel lensing technique. In Sects. 4 and 5 we present maps of optical depth, event rate and typical event time duration, addressing the modification with respect to classical microlensing values, due to the influence of the threshold magnification in pixel lensing searches. Finally in Sect. 6 we present some conclusions.

\section{Source and lens models}

The M 31 disk, bulge and halo mass distributions are described adopting the parameters of the Reference model in Kerins (2004), which provides remarkably good fits to the M31 surface brightness and rotation curve profiles.

This model, by using an average set of parameter values less extreme with respect to the massive halo, massive bulge and massive disk models in Table 1, can be considered a more likely candidate model for the mass distributions in the M 31 galaxy.

Accordingly, the mass density of the M 31 disk stars is described by a sech-squared profile

$\rho_{\mathrm{D}}(R, z)=\rho_{\mathrm{D}}(0) \exp (-R / h) \operatorname{sech}^{2}(z / H)$ 
where $R$ is the distance on the $\mathrm{M} 31$ disk plane, $H \simeq 0.3 \mathrm{kpc}$ and $h \simeq 6.4 \mathrm{kpc}$ are, respectively, the scale height and scale lengths of the disk and $\rho_{\mathrm{D}}(0) \simeq 3.5 \times 10^{8} M_{\odot} \mathrm{kpc}^{-3}$ is the central mass density. The disk is truncated at $R \simeq 40 \mathrm{kpc}$ so that the total mass is $5.3 \times 10^{10} M_{\odot}$.

As usual, the M 31 disk is assumed to be inclined at the angle $i=77^{\circ}$ and the azimuthal angle relative to the near minor axis $\phi=-38.6^{\circ}$.

The M 31 bulge is parameterized by a flattened power law of the form

$\rho_{\mathrm{B}}(R, z)=\rho_{\mathrm{B}}(0)\left[1+\left(\frac{R}{a}\right)^{2}+q^{-2}\left(\frac{z}{a}\right)^{2}\right]^{-s / 2}$,

where the coordinates $x$ and $y$ span the M 31 disk plane ( $z$ is perpendicular to it), $\rho_{\mathrm{B}}(0) \simeq 4.5 \times 10^{9} M_{\odot} \mathrm{kpc}^{-3}, q \simeq 0.6$ is the ratio of the minor to major axes, $a \simeq 1 \mathrm{kpc}$ and $s \simeq 3.8$ (Reitzel et al. 1998). The bulge is truncated at $40 \mathrm{kpc}$ and its total mass is $\simeq 4.4 \times 10^{10} M_{\odot}$.

The dark matter in the M 31 halo is assumed to follow an isothermal profile

$\rho_{\mathrm{H}}(r)=\rho_{\mathrm{H}}(0) \frac{a^{2}}{a^{2}+r^{2}}$,

with core radius $a \simeq 4 \mathrm{kpc}$, and central dark matter density $\rho_{\mathrm{H}}(0) \simeq 6.5 \times 10^{7} M_{\odot} \mathrm{kpc}^{-3}$, so that the total rotational velocity in the M 31 halo is $v_{\text {rot }} \simeq 235 \mathrm{~km} \mathrm{~s}^{-1}$. The M 31 halo is truncated at $R \simeq 100 \mathrm{kpc}$ and the total dark mass within this distance is $\simeq 1.23 \times 10^{12} M_{\odot}$.

As usual, the mass density profile for a MW disk is described with a double exponential profile

$\rho_{\mathrm{D}}(R, z)=\rho_{\mathrm{D}}\left(R_{0}\right) \exp \left(-\left(R-R_{0}\right) / h\right) \exp (-|z| / H)$,

with the Earth's position from the Galactic center at $R_{0} \simeq$ $8.5 \mathrm{kpc}$, scale height $H \simeq 0.3 \mathrm{kpc}$, scale length $h \simeq 3.5 \mathrm{kpc}$ and local mass density $\rho_{\mathrm{D}}\left(R_{0}\right) \simeq 1.67 \times 10^{8} M_{\odot} \mathrm{kpc}^{-3}$.

The MW bulge ${ }^{1}$ is described by the triaxial bulge model with mass density profile (Dwek et al. 1995)

$\rho_{\mathrm{B}}(x, y, z)=\frac{M_{\mathrm{B}}}{8 \pi a b c} \mathrm{e}^{-s^{2} / 2}$,

where $s^{4}=\left[(x / a)^{2}+(y / b)^{2}\right]^{2}+(z / c)^{4}$, the bulge mass is $M_{\mathrm{B}} \simeq 2 \times 10^{10} M_{\odot}$ and the scale lengths are $a \simeq 1.49 \mathrm{kpc}$, $b \simeq 0.58 \mathrm{kpc}$ and $c \simeq 0.40 \mathrm{kpc}$. Here, the coordinates $x$ and $y$ span the Galactic disk plane, whereas $z$ is perpendicular to it.

The dark halo in our Galaxy is also assumed to follow an isothermal profile with core radius $a \simeq 5.6 \mathrm{kpc}$ and local dark matter density $\rho_{\mathrm{H}}\left(R_{0}\right) \simeq 1.09 \times 10^{7} M_{\odot} \mathrm{kpc}^{-3}$. The corresponding total asymptotic rotational velocity is $v_{\text {rot }} \simeq 220 \mathrm{~km} \mathrm{~s}^{-1}$. The MW halo is truncated at $R \simeq 100 \mathrm{kpc}$ and the dark mass within this distance is $\simeq 1.30 \times 10^{12} M_{\odot}$.

For both M 31 and MW halos, the fraction of dark matter in form of MACHOs is assumed to be $f_{\mathrm{MACHO}} \simeq 0.2$ (Alcock et al. 2000).

Moreover, as usual, we assume the random velocities of stars and MACHOs to follow Maxwellian distributions with

1 Although it does not contribute to microlensing towards M 31, it contributes to the dynamical mass of the Galaxy. one-dimensional velocity dispersion $\sigma=30,100,166 \mathrm{~km} \mathrm{~s}^{-1}$ and $30,156 \mathrm{~km} \mathrm{~s}^{-1}$ for the M31 disk, bulge, halo and MW disk and halo, respectively (see also Kerins et al. 2001; An et al. 2004). In addition an M 31 bulge rotational velocity of $30 \mathrm{~km} \mathrm{~s}^{-1}$ is assumed.

\section{Pixel lensing basics}

The main difference between gravitational microlensing and pixel lensing observations relies in the fact that in pixel lensing a large number of stars contribute to the same pixel and therefore only bright and sufficiently magnified sources can be identified as microlensing events. In pixel lensing analysis one usually defines a minimum amplification that depends on the baseline photon counts (Ansari et al. 1997)

$N_{\mathrm{bl}}=N_{\mathrm{gal}}+N_{\mathrm{sky}}$,

which is the sum of the M31 surface brightness and sky contribution.

The excess photon counts per pixel due to an ongoing microlensing event is

$\Delta N_{\text {pix }}=N_{\text {bl }}\left[A_{\text {pix }}-1\right]=f_{\text {see }} N_{\mathrm{s}}[A(t)-1]$

where $N_{\mathrm{s}}$ and $N_{\mathrm{bl}}$ are the source and baseline photon counts in the absence of lensing, $A(t)$ is the source magnification factor due to lensing and $f_{\text {see }}$ is the fraction of the seeing disk contained in a pixel.

As usual, the amplification factor is given by (see, e.g., Griest 1991, and references therein)

$A(u)=\frac{u^{2}+2}{\sqrt{u^{2}\left(u^{2}+4\right)}}$,

where

$u(t)^{2}=u_{0}^{2}+\left(\frac{t-t_{0}}{t_{\mathrm{E}}}\right)^{2}$

is the impact distance in units of the Einstein radius

$R_{\mathrm{E}}=\left[\left(4 G m_{1} / c^{2}\right) D_{\mathrm{l}}\left(D_{\mathrm{s}}-D_{\mathrm{l}}\right) / D_{\mathrm{s}}\right]^{1 / 2}$,

and $u_{0}$ is the impact parameter in units of $R_{\mathrm{E}}$. Moreover, $t_{\mathrm{E}}=$ $2 R_{\mathrm{E}} / v_{\perp}$ is the Einstein time, $t_{0}$ the time of maximum magnification, $D_{\mathrm{s}}$ and $D_{1}$ are the source and lens distances from the observer and $v_{\perp}$ is the lens transverse velocity with respect to the line of sight.

The number of photons in a pixel is given by

$N_{\text {pix }}=\Delta N_{\text {pix }}+N_{\text {bl }}$

and a pixel lensing event will be detectable if the excess pixel photon counts $\Delta N_{\text {pix }}$ are greater than the pixel noise

$\sigma=\max \left(\sigma_{\mathrm{T}}, \sqrt{N_{\text {pix }}}\right)$,

$\sigma_{\mathrm{T}}$ being the minimum noise level determined by the pixel flux stability and $\sqrt{N_{\text {pix }}}$ the statistical photon fluctuation. 
By regarding a signal as being statistically significant if it occurs at a level $3 \sigma$ above the baseline counts $N_{\mathrm{bl}}$, one obtains $A_{\min } \geq 1+3 \sigma /\left(f_{\mathrm{see}} N_{\mathrm{s}}\right)$. If $\sigma$ is taken equal to the minimum noise level $\sigma_{\mathrm{T}}$, the obtained threshold magnification $A_{\mathrm{T}}$ is (Kerins et al. 2001)

$A_{\mathrm{T}}=1+0.0075 \frac{N_{\mathrm{bl}}}{f_{\mathrm{see}} N_{\mathrm{s}}}$

which corresponds to a threshold value $u_{\mathrm{T}}$ for the impact distance via the relation in Eq. (8). As one can see, $u_{\mathrm{T}}$ depends on the source magnitude $M$, the line of sight to M 31 and the observing conditions.

\section{Pixel lensing optical depth and event rate}

In pixel lensing analysis, the effect of the existence of the threshold magnification (or, equivalently, of a threshold value of the impact parameter) is usually taken into account in estimating the pixel lensing rate (Kerins et al. 2001, 2003)

$\Gamma_{\mathrm{p}}(x, y)=\left\langle u_{\mathrm{T}}(x, y)\right\rangle_{\phi} \Gamma_{\mathrm{c}}(x, y)$,

where $x$ and $y$ are coordinates in the plane orthogonal to the line of sight and $\left\langle u_{\mathrm{T}}(x, y)\right\rangle_{\phi}$ is averaged on the source magnitude $M$, namely

$\left\langle u_{\mathrm{T}}(x, y)\right\rangle_{\phi}=\frac{\int_{M_{1}}^{M_{2}} u_{\mathrm{T}}(M ; x, y) \phi(M) \mathrm{d} M}{\int_{M_{1}}^{M_{2}} \phi(M) \mathrm{d} M}$,

$M_{1}-M_{2}$ (to be specified below) being the limiting values for the source magnitude and $\phi(M) \mathrm{d} M$ the luminosity function, i.e. the number density of sources in the absolute magnitude interval $(M, M+\mathrm{d} M)$.

Pixel lensing event detection is usually performed in $R$ or $I$ bands in order to minimize light absorption by the intervening dust in M 31 and MW disks. Indeed, these bands offer the best compromise between sampling and sky background, while other bands ( $B$ and $V$ ) are commonly used to test achromaticity of the candidate events.

In the present analysis, as reference values, we adopt the parameters of the Sloan-r filter on the Wide-Field Camera of the Isaac Newton Telescope (Kerins et al. 2001). Therefore, since the red giants are the most luminous stars in the red band, we may safely assume that the overwhelming majority of the pixel lensing event sources are red giants. Moreover, gives the lack of precise information about the stellar luminosity function in the M 31 galaxy, we assume that the same function $\phi(M)$ holds both for the Galaxy and M 31 and does not depend on position.

Accordingly, in the range of magnitude $-6 \leq M \leq 16$ the stellar luminosity function is proportional to the following expression (Mamon \& Soneira 1982)

$\phi(M) \propto \frac{10^{\beta\left(M-M^{*}\right)}}{\left[1+10^{-(\alpha-\beta) \delta\left(M-M^{*}\right)}\right]^{1 / \delta}}$,

where, in the red band, $M^{*}=1.4, \alpha \simeq 0.74, \beta=0.045$ and $\delta=1 / 3$.
On the other hand, the fraction of red giants (over the total star number) as a function of $M$ can be approximated as (Mamon \& Soneira 1982)

$$
\begin{aligned}
& f_{\mathrm{RG}}(M)=1-C \exp \left[\alpha(M+\beta)^{\gamma}\right] \text { for }-6 \leq M \leq 3 \\
& =0 \quad \text { for } M \geq 3 \text {, }
\end{aligned}
$$

where, in the red band, $C \simeq 0.31, \alpha \simeq 6.5 \times 10^{-4}, \beta=7.5$ and $\gamma \simeq 3$.2. Therefore, the fraction of red giants averaged over the magnitude is given by

$\left\langle f_{\mathrm{RG}}\right\rangle=\frac{\int_{-6}^{3} \phi(M) f_{\mathrm{RG}}(M) \mathrm{d} M}{\int_{-6}^{16} \phi(M) \mathrm{d} M}$,

from which we obtain $\left\langle f_{\mathrm{RG}}\right\rangle \simeq 5.3 \times 10^{-3}$.

Averaging the pixel lensing rate in Eq. (14) on the source density we obtain

$\left\langle\Gamma_{\mathrm{p}}(x, y)\right\rangle=\left\langle u_{\mathrm{T}}(x, y)\right\rangle_{\phi}\left\langle\Gamma_{\mathrm{c}}(x, y)\right\rangle_{n_{\mathrm{s}}}$,

where the mean classical rate $\left\langle\Gamma_{\mathrm{c}}(x, y)\right\rangle_{n_{\mathrm{s}}}$ is

$\left\langle\Gamma_{\mathrm{c}}(x, y)\right\rangle_{n_{\mathrm{s}}}=\frac{\int \Gamma_{\mathrm{c}}\left(D_{\mathrm{s}} ; x, y\right) n_{\mathrm{s}}\left(D_{\mathrm{s}} ; x, y\right) \mathrm{d} D_{\mathrm{s}}}{\int n_{\mathrm{s}}\left(D_{\mathrm{s}} ; x, y\right) \mathrm{d} D_{\mathrm{s}}}$.

In turn, for a fixed source distance, $\Gamma_{\mathrm{c}}\left(D_{\mathrm{s}} ; x, y\right)$ is obtained by the classical microlensing rate for a lens of mass $m_{1}$ (Griest 1991), by averaging on the lens mass, namely

$\Gamma_{\mathrm{c}}\left(D_{\mathrm{s}} ; x, y\right)=\frac{\int \Gamma_{\mathrm{c}}\left(m_{1} ; D_{\mathrm{s}}, x, y\right) P\left(m_{1}\right) \mathrm{d} m_{1}}{\int P\left(m_{1}\right) \mathrm{d} m_{1}}$,

where $P\left(m_{1}\right)$ is the lens mass distribution function.

For lenses belonging to the bulge and disk star populations, lenses are assumed to follow a broken power law (see e.g. An et al. 2004, and references therein)

$$
\begin{aligned}
P\left(m_{1}\right) & \propto m_{1}^{-1.4} \text { for } 0.1 M_{\odot} \leq m_{1} \leq 0.5 M_{\odot} \\
& \propto m_{1}^{-2.2} \text { for } 0.5 M_{\odot} \leq m_{1} \leq\left(m_{1}\right)_{\mathrm{up}}
\end{aligned}
$$

where the upper limit $\left(m_{1}\right)_{\text {up }}$ is $1 M_{\odot}$ for M 31 bulge stars and $10 M_{\odot}$ for M31 and MW disk stars. The resulting mean mass for lenses in the bulges and disks are $\left\langle m_{\mathrm{b}}\right\rangle \sim 0.31 M_{\odot}$ and $\left\langle m_{\mathrm{d}}\right\rangle \sim 0.53 M_{\odot}$, respectively.

For the lens mass in the M 31 and MW halos we assume the $\delta$-function approximation and we take a MACHO mass $m_{\mathrm{MACHO}} \simeq 0.5 M_{\odot}$, according to the mean value in the analysis of microlensing data towards LMC (Alcock et al. 2000).

As usual, the mean number of expected events in classical microlensing $\left\langle E_{\mathrm{c}}(x, y)\right\rangle$ and pixel lensing $\left\langle E_{\mathrm{p}}(x, y)\right\rangle$, respectively, are related to the observation time $t_{\mathrm{obs}}$, source column density $N_{\mathrm{S}}$ and mean fraction of red giants $\left\langle f_{\mathrm{RG}}\right\rangle$ by

$\left\langle E_{\mathrm{c}}(x, y)\right\rangle=\left\langle\Gamma_{\mathrm{c}}(x, y)\right\rangle_{n_{\mathrm{s}}} t_{\mathrm{obs}} N_{\mathrm{s}}(x, y)\left\langle f_{\mathrm{RG}}\right\rangle$,

$\left\langle E_{\mathrm{p}}(x, y)\right\rangle=\left\langle\Gamma_{\mathrm{p}}(x, y)\right\rangle_{n_{\mathrm{s}}} t_{\mathrm{obs}} N_{\mathrm{s}}(x, y)\left\langle f_{\mathrm{RG}}\right\rangle$,

where the source column density is

$N_{\mathrm{s}}(x, y)=\int n_{\mathrm{s}}\left(D_{\mathrm{s}} ; x, y\right) \mathrm{d} D_{\mathrm{s}}$. 
However, the classical microlensing rate depends on several source and lens parameters, in particular on the lens mass and transverse velocity of the source and lens. Therefore, due to the parameter degeneracy, it does not give precise information on the lens population, at least in the M31 regions where microlensing by stars in M31 itself (self-lensing) and by MACHOs in M 31 and MW halos (dark lensing) occur with comparable probability. Indeed, as usual, the probability for a given lens population is

$P_{1}=\frac{\sum_{\mathrm{s}} \Gamma_{\mathrm{sl}}}{\sum_{\mathrm{s}} \sum_{1} \Gamma_{\mathrm{sl}}}$

On the other hand, the classical microlensing optical depth

$\tau_{\mathrm{c}}\left(D_{\mathrm{s}} ; x, y\right)=\int_{0}^{D_{\mathrm{s}}} \pi R_{\mathrm{E}}^{2} n_{\mathrm{l}}\left(D_{1}\right) \mathrm{d} D_{\mathrm{l}}$

is a geometrical quantity and depends on a small number of parameters and can be used as in Eq. (26) to determine the lens nature.

Physically the optical depth is the number of ongoing microlensing events per source star at any instant in time. So, one can also compute the instantaneous event number density, as a function of position, by multiplying the optical depth by the number density of sources

$\left\langle I E_{\mathrm{c}}(x, y)\right\rangle=\left\langle\tau_{\mathrm{c}}(x, y)\right\rangle_{n_{\mathrm{s}}} N_{\mathrm{s}}(x, y)\left\langle f_{\mathrm{RG}}\right\rangle$.

However, Eq. (27) is the usual definition for the optical depth in classical gravitational microlensing, while in the case of pixel lensing it is necessary to take into account the effect of $u_{\mathrm{T}}(M ; x, y)$.

In order to generalize the $\tau$ definition to the pixel lensing case, a new definition (which joins the advantage of using a geometrical quantity with the main characteristic of the pixel lensing technique, i.e. the effect of the baseline) is introduced (see also Kerins 2004)

$\left\langle\tau_{\mathrm{p}}(x, y)\right\rangle=\left\langle u_{\mathrm{T}}^{2}(x, y)\right\rangle_{\phi}\left\langle\tau_{\mathrm{c}}(x, y)\right\rangle_{n_{\mathrm{s}}}$,

where $\left\langle\tau_{\mathrm{c}}(x, y)\right\rangle_{n_{\mathrm{s}}}$ is

$\left\langle\tau_{\mathrm{c}}(x, y)\right\rangle_{n_{\mathrm{s}}}=\frac{\int \tau_{\mathrm{c}}(x, y) n_{\mathrm{s}}\left(D_{\mathrm{s}} ; x, y\right) \mathrm{d} D_{\mathrm{s}}}{\int n_{\mathrm{s}}\left(D_{\mathrm{s}} ; x, y\right) \mathrm{d} D_{\mathrm{s}}}$.

The factor $\left\langle u_{\mathrm{T}}^{2}(x, y)\right\rangle_{\phi}$ in Eq. (29) comes from the consideration that the Einstein radius $R_{\mathrm{E}}$, which enters quadratically in $\tau_{\mathrm{c}}$, has to be multiplied by $u_{\mathrm{T}}$ (always less than unity for pixel lensing).

Accordingly, the instantaneous event number density in pixel lensing is given by

$\left\langle I E_{\mathrm{p}}(x, y)\right\rangle=\left\langle\tau_{\mathrm{p}}(x, y)\right\rangle_{n_{\mathrm{s}}} N_{\mathrm{s}}(x, y)\left\langle f_{\mathrm{RG}}\right\rangle$.

Here we note that in evaluating $\left\langle E_{\mathrm{p}}(x, y)\right\rangle$ for each model considered in Table 1, we have to take into account that the number of detectable pixel lensing events does not depends on the typical source luminosity $L_{\mathrm{s}}$ to first order (Kerins 2004). Indeed, although for a fixed source luminosity $L_{\mathrm{s}}$ the number of sources
$N_{\mathrm{s}} \propto L_{\mathrm{s}}^{-1}$, the pixel lensing rate per source $\Gamma_{\mathrm{p}} \propto L_{\mathrm{s}},{ }^{2}$ so that the event number does not depend on $L_{\mathrm{s}}$. The same also holds for the instantaneous event number density $\left\langle I E_{\mathrm{p}}(x, y)\right\rangle$.

\section{Pixel lensing event duration}

In pixel microlensing, due to the large number of stars simultaneously contributing to the same pixel, the flux from a single star in the absence of lensing is generally not observable. Thus, the Einstein time $t_{\mathrm{E}}$ cannot be determined reliably by fitting the observed light curve.

Indeed, another estimator of the event time duration has been proposed, namely the full-width half-maximum event duration $t_{F W H M}$, which depends on $t_{\mathrm{E}}$ and $u_{0}$ (Gondolo 1999)

$t_{F W H M}=t_{\mathrm{E}} w\left(u_{0}\right)$,

where $w\left(u_{0}\right)$ is given by

$w\left(u_{0}\right)=2 \sqrt{2 f\left[f\left(u_{0}^{2}\right)\right]-u_{0}^{2}}$

and $f(x)=A(x)-1$, where $A(x)$ is the amplification factor in Eq. (8).

This quantity can be put in a different form (Kerins et al. 2001)

$t_{F W H M}=2 \sqrt{2} t_{\mathrm{E}}\left(\frac{a+2}{\sqrt{a^{2}+4 a}}-\frac{a+1}{\sqrt{a^{2}+2 a}}\right)^{1 / 2}$,

where $a=A_{\max }-1$.

In the limit of large amplification $A_{\max } \gg 1$ (or, equivalently, $u_{0} \ll 1$ ) one obtains

$a \simeq \frac{3 u_{0}}{8}+\frac{1}{u_{0}}-1+O\left(u_{0}^{3}\right)$.

Using Eqs. (35) in (34), the full-width half-maximum event duration can be approximated by

$t_{F W H M} \simeq 2 \sqrt{2} t_{\mathrm{E}} u_{0}\left(1-u_{0}\right)+O\left(u_{0}^{3}\right)$.

Clearly, while in classical microlensing $u_{0}$ may be determined, in pixel microlensing the background overcomes the source baseline making $u_{0}$ unknown, implying that an average procedure on $u_{0}$ is needed to estimate the mean event duration. Since the impact parameter $u_{0}$ varies in the range $\left(0, u_{\mathrm{T}}\right)$ and the probability of $u_{0}$ being in the range $u_{0}-u_{0}+\mathrm{d} u_{0}$ is $P\left(u_{0}\right) \mathrm{d} u_{0} \propto 2 \pi u_{0} \mathrm{~d} u_{0}$ (the area of the circular ring of radius $u_{0}$ and thickness $\mathrm{d} u_{0}$ ), by averaging $t_{F W H M}$ on the impact parameter, in the limit of large amplification, one gets

$\left\langle t_{F W H M}\right\rangle_{u_{0}} \simeq 2 \sqrt{2} t_{\mathrm{E}} \frac{\int_{0}^{u_{\mathrm{T}}} u_{0}^{2}\left(1-u_{0}\right) \mathrm{d} u_{0}}{\int_{0}^{u_{\mathrm{T}}} u_{0} \mathrm{~d} u_{0}} \simeq u_{\mathrm{T}} t_{\mathrm{c}}$,

where

$t_{\mathrm{c}}=\frac{4 \sqrt{2}}{3} t_{\mathrm{E}}(x, y)$.

${ }^{2}$ Indeed, the faintest detectable pixel lensing events require a threshold source magnification $A_{\mathrm{T}} \propto L_{\mathrm{s}}^{-1}$, to be seen against the local background. On the other hand, in pixel lensing the bulk of observed events involves highly magnified sources for which $A_{\mathrm{T}} \propto u_{\mathrm{T}}^{-1}$. Therefore since $\Gamma_{\mathrm{p}} \propto u_{\mathrm{T}}$, it follows that $\Gamma_{\mathrm{p}} \propto L_{\mathrm{s}}$. 
Inspection of the relation in Eq. (37) and of Eqs. (14), (19) and (29), lead us to introduce for pixel lensing a new event time scale estimator $\left\langle t_{\mathrm{p}}(x, y)\right\rangle$ defined as

$\left\langle t_{\mathrm{p}}(x, y)\right\rangle=\left\langle u_{\mathrm{T}}(x, y)\right\rangle_{\phi}\left\langle t_{\mathrm{c}}(x, y)\right\rangle_{n_{\mathrm{s}}}$,

where

$\left\langle t_{\mathrm{c}}(x, y)\right\rangle_{n_{\mathrm{s}}}=\frac{\int t_{\mathrm{c}}(x, y) n_{\mathrm{s}}\left(D_{\mathrm{s}} ; x, y\right) \mathrm{d} D_{\mathrm{s}}}{\int n_{\mathrm{s}}\left(D_{\mathrm{s}} ; x, y\right) \mathrm{d} D_{\mathrm{s}}}$,

and

$t_{\mathrm{c}}(x, y)=\frac{4 \sqrt{2}}{3} \frac{\int t_{\mathrm{E}}(x, y) \mathrm{d} \Gamma(x, y)}{\Gamma(x, y)}$.

Clearly, the pixel lensing time scale $\left\langle t_{\mathrm{p}}(x, y)\right\rangle$ turns out to be the full-width half-maximum event duration averaged over the impact parameter.

\section{Results}

Before discussing the results obtained, we summarize some assumptions used in the present analysis. First, we assume perfect sensitivity to pixel lensing event detection in M31 pixel lensing searches. Moreover, as reference values, we use the parameters for the Isaac Newton Telescope and WFC (Wide-Field Camera) adopted by the POINT-AGAPE collaboration (Kerins et al. 2001, 2003).

The Telescope diameter, the pixel field of view and the image exposition time are $2.5 \mathrm{~m}, 0.33$ arcsec and $t_{\exp }=760 \mathrm{~s}$, respectively. We also assume a gain or conversion factor of $2.8 \mathrm{e}^{-} / \mathrm{ADU}$, and a loss factor $\simeq 3$, both for atmospheric and instrumental effects. The zero-point with the Sloan-r WFC is $\sim 24.3$ mag $\operatorname{arcsec}^{-2}$.

To take into account the effect of seeing, we employ an analysis based on superpixel photometry. Adopting a value of 2.4 arcsec for the worst seeing value, we take a superpixel dimension of $7 \times 7$ pixel and adopt a minimum noise level of $\sigma_{\mathrm{T}} \sim 2.5 \times 10^{-3} N_{\mathrm{bl}}$. We also assume that typically 87 per cent of a point spread function (PSF) positioned at the center of a superpixel is contained within the superpixel itself.

The considered sky background is $m_{\text {sky }} \simeq$ 20.9 mag $\operatorname{arcsec}^{-2}$ (corresponding to a Moon eclipse), so that the typical sky luminosity is $N_{\text {sky }} \sim 1600$ counts/pixel, which enters in the baseline count estimates. However, for comparison purposes with Kerins (2004), some results in Tables 3-5 are also given for a sky background $m_{\text {sky }} \simeq 19.5 \mathrm{mag} \mathrm{arcsec}^{-2}$ and $f_{\text {see }}=0.40$ (corresponding to a randomly positioned PSF within the superpixel).

Moreover, all the figures presented in Sect. 6 are given for the Reference model (see Table 1). The effect of varying the model parameters for the M31 bulge, disk and halo is also shown in Tables 3-5.

Finally, we recall that $N_{\mathrm{bl}}(x, y)$ is obtained from Eq. (6) where $N_{\text {gal }}(x, y)$ follows from the M31 photometry given by Kent (1989).

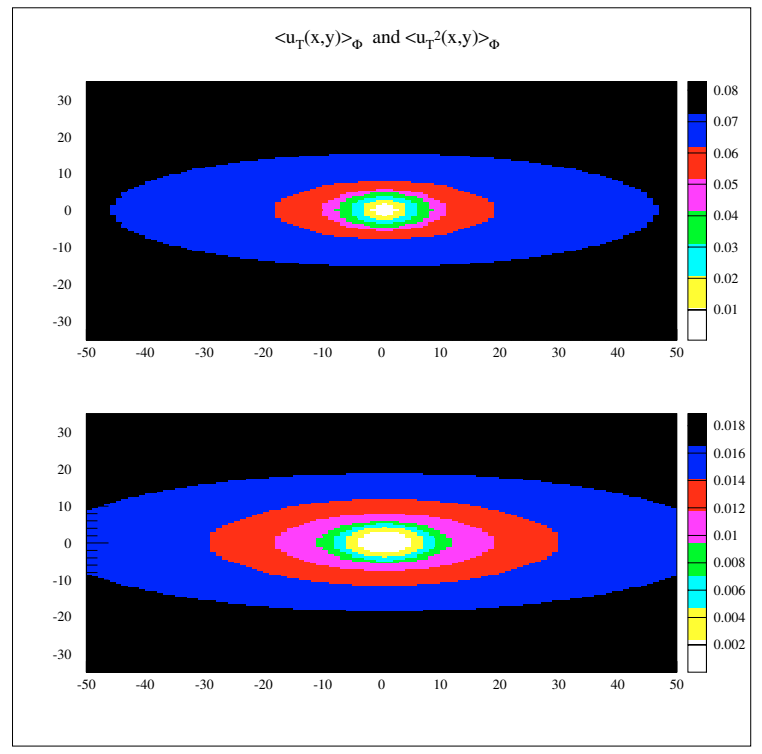

Fig. 1. The mean impact parameter maps $\left\langle u_{\mathrm{T}}(x, y)\right\rangle_{\phi}$ and $\left\langle u_{\mathrm{T}}^{2}(x, y)\right\rangle_{\phi}$ towards M 31 are given, for selected directions towards M 31 corresponding to different $(x, y)$ coordinates (in units of arcmin) centered on M 31 and aligned along the major and minor axes of the projected light profile.

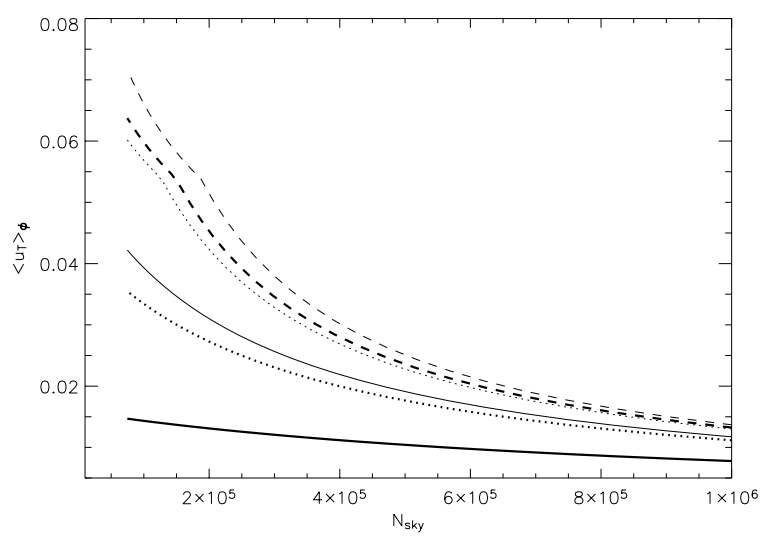

Fig. 2. The mean impact parameter $\left\langle u_{\mathrm{T}}(x, y)\right\rangle_{\phi}$ is given as a function of the background photon counts $N_{\text {sky }}$ in a pixel, for selected directions towards M 31 corresponding to different $(x, y)$ coordinates (in units of arcmin) in the sky plane. Thin lines, from the bottom to the upper part of the figure, refer to $(8,0),(16,0)$ and $(32,0)$; thick lines are for $(0,-2),(0,-4)$ and $(0,-8)$ coordinates, respectively.

\subsection{Threshold impact parameter}

In Fig. 1 maps of the mean threshold impact parameter $\left\langle u_{\mathrm{T}}(x, y)\right\rangle_{\phi}$ and $\left\langle u_{\mathrm{T}}^{2}(x, y)\right\rangle_{\phi}$ towards M 31 are shown.

In this and following figures we use Cartesian coordinates $x$ and $y$ centered on M 31 and aligned along the major and minor axes of the projected light profile, respectively.

As one can see in Fig. 1, the effect of the higher luminosity of the inner region of M 31 with respect to the outer part of the galaxy is to reduce the obtained $\left\langle u_{\mathrm{T}}(x, y)\right\rangle_{\phi}$ values by about an order of magnitude.

In Fig. 2, for selected lines of sight to M31, we show how $\left\langle u_{\mathrm{T}}(x, y)\right\rangle_{\phi}$ depends on the photon counts $N_{\text {sky }}$ from the 
Table 2. The threshold impact parameters $\left\langle u_{\mathrm{T}}\right\rangle_{\phi}$ and $\left\langle u_{\mathrm{T}}^{2}\right\rangle_{\phi}$ averaged over the whole M 31 galaxy are given for different values of the superpixel dimension $N \times N$, sky background $m_{\text {sky }}$, fraction $f_{\text {see }}$ of the superpixel covered by the PSF and superpixel flux stability $\sigma_{\mathrm{T}}$.

\begin{tabular}{cccccc}
\hline \hline$N \times N$ & $\begin{array}{c}m_{\text {sky }} \\
\left(\mathrm{mag} \mathrm{arcsec}^{-2}\right)\end{array}$ & $f_{\text {see }}$ & $\sigma_{\mathrm{T}}$ & $\left\langle u_{\mathrm{T}}\right\rangle_{\phi}$ & $\left\langle u_{\mathrm{T}}^{2}\right\rangle_{\phi}$ \\
\hline $3 \times 3$ & 20.9 & 0.87 & $2.5 \times 10^{-3} \mathrm{~N}_{\mathrm{bl}}$ & $1.10 \times 10^{-1}$ & $3.17 \times 10^{-2}$ \\
$5 \times 5$ & 20.9 & 0.87 & $2.5 \times 10^{-3} \mathrm{~N}_{\mathrm{bl}}$ & $7.11 \times 10^{-2}$ & $1.59 \times 10^{-2}$ \\
$7 \times 7$ & 20.9 & 0.87 & $2.5 \times 10^{-3} \mathrm{~N}_{\mathrm{bl}}$ & $5.10 \times 10^{-2}$ & $9.56 \times 10^{-3}$ \\
$7 \times 7$ & 20.9 & 0.40 & $2.5 \times 10^{-3} \mathrm{~N}_{\mathrm{bl}}$ & $2.57 \times 10^{-2}$ & $3.13 \times 10^{-3}$ \\
$7 \times 7$ & 19.5 & 0.40 & $2.5 \times 10^{-3} \mathrm{~N}_{\mathrm{bl}}$ & $1.44 \times 10^{-2}$ & $1.17 \times 10^{-3}$ \\
$7 \times 7$ & 19.5 & 0.40 & $1.0 \times 10^{-2} \mathrm{~N}_{\mathrm{bl}}$ & $3.82 \times 10^{-3}$ & $1.23 \times 10^{-4}$ \\
\hline
\end{tabular}

Table 3. The expected number of events $\left\langle E_{\mathrm{p}}\right\rangle$ per year in pixel lensing observations towards the M 31 galaxy for different locations of sources and lenses is shown. We consider the $100 \times 70 \mathrm{arcmin}^{2}$ region oriented along the major axis of M31. and exclude events occurring within a radius of 8 arcmin of the M 31 center. Sources and lenses in the M 31 bulge and disk are indicated by indices 1 and 2, while lenses in the M 31 halo and MW disk and halo by indices 3,5 and 6. The first and second indices refer to source and lens, respectively. The mean mass of bulge and disk stars is $\sim 0.31 M_{\odot}$ and $\sim 0.53 M_{\odot}$, respectively. For the lenses in the M31 and MW halos we take a mass of $\simeq 0.5 M_{\odot}$ and a MACHO fraction $f_{\text {MACHO }} \simeq 20 \%$.

\begin{tabular}{ccccccccccccc}
\hline \hline & & 11 & 12 & 13 & 15 & 16 & 21 & 22 & 23 & 25 & 26 & Overall \\
\hline Model & $\left\langle u_{\mathrm{T}}\right\rangle_{\phi}$ & & & & & & & & & & & \\
\hline Massive halo & $5.10 \times 10^{-2}$ & 11.9 & 16.0 & 10.7 & 0.8 & 4.2 & 15.9 & 11.7 & 25.4 & 1.7 & 9.3 & 107.5 \\
Massive bulge & & 57.9 & 100.9 & 10.4 & 1.6 & 9.0 & 20.8 & 20.5 & 8.4 & 1.1 & 5.9 & 236.4 \\
Massive disk & & 11.8 & 92.8 & 3.7 & 0.7 & 4.2 & 16.1 & 69.3 & 10.2 & 1.7 & 9.4 & 219.9 \\
Reference & & 12.5 & 26.4 & 8.9 & 0.8 & 4.4 & 13.4 & 16.0 & 19.5 & 1.5 & 8.3 & 111.8 \\
\hline Reference & $1.4 \times 10^{-2}$ & 3.5 & 7.2 & 2.4 & 0.2 & 1.2 & 3.6 & 4.3 & 5.2 & 0.4 & 2.2 & 30.4 \\
\hline
\end{tabular}

Table 4. The same as in Table 3 for lenses located in the M 31 galaxy. In the last three columns we give the calculated pixel event number for the South/North Semisphere and in brackets their ratio.

\begin{tabular}{ccccccccc}
\hline \hline Model & $\left\langle u_{\mathrm{T}}\right\rangle_{\phi}$ & Bulge & Disk & M31 halo & M31 overall & Stellar & M31 halo & 23 \\
\hline Massive halo & \multirow{2}{*}{$5.10 \times 10^{-2}$} & 27.7 & 27.7 & 36.1 & 91.5 & $31.4 / 24.0(1.3)$ & $25.3 / 10.8(2.3)$ & $19.6 / 5.8(3.4)$ \\
Massive bulge & & 78.6 & 121.4 & 18.7 & 218.7 & $72.6 / 127.4(0.6)$ & $11.3 / 7.4(1.5)$ & $6.0 / 2.4(2.5)$ \\
Massive disk & & 27.9 & 162.0 & 13.9 & 203.8 & $85.5 / 104.5(0.8)$ & $9.2 / 4.7(1.9)$ & $7.2 / 2.9(2.5)$ \\
Reference & & 25.9 & 42.4 & 28.4 & 96.7 & $33.9 / 34.4(1.0)$ & $19.6 / 8.9(2.2)$ & $14.9 / 4.6(3.2)$ \\
\hline Reference & $1.4 \times 10^{-2}$ & 7.1 & 11.5 & 7.7 & 26.3 & $9.3 / 9.4(1.0)$ & $5.3 / 2.4(2.2)$ & $4.0 / 1.2(3.2)$ \\
\hline
\end{tabular}

background, which is approximated as a diffuse source of magnitude $m_{\text {sky }}$ in the range 20.9-18.9 mag arcsec ${ }^{-2}$. In Fig. 2 we consider several lines of sight to M 31 with different $(x, y)$ coordinates (in units of arcmin) in the orthogonal plane. Thin lines, from the bottom to the top, refer to $(0,-0.2),(4,-0.2)$ and $(8,-0.2)$, thick lines are for $(0,-2),(4,-2)$ and $(8,-2)$ coordinates. It is evident that $\left\langle u_{\mathrm{T}}(x, y)\right\rangle_{\phi}$ weakly depends on $N_{\text {sky }}$ in the inner M 31 regions, where $N_{\mathrm{bl}}$ is dominated by the counts $N_{\text {gal }}$ from the M 31 surface brightness. Moreover, for a fixed number of counts $N_{\text {sky }}$ from the sky, $\left\langle u_{\mathrm{T}}(x, y)\right\rangle_{\phi}$ decreases with increasing $N_{\text {gal }}$.

We note that by averaging $\left\langle u_{\mathrm{T}}(x, y)\right\rangle_{\phi}$ and $\left\langle u_{\mathrm{T}}^{2}(x, y)\right\rangle_{\phi}$ (weighting with the star number density) on the whole field of view towards the M31 galaxy, we obtain $\left\langle u_{\mathrm{T}}\right\rangle_{\phi} \simeq 5.10 \times 10^{-2}$ and $\left\langle u_{\mathrm{T}}^{2}\right\rangle_{\phi} \simeq 8.8 \times 10^{-3}$.

In Table 2 the effect on $\left\langle u_{\mathrm{T}}(x, y)\right\rangle_{\phi}$ and $\left\langle u_{\mathrm{T}}^{2}(x, y)\right\rangle_{\phi}$ of changing the parameter values for the superpixel dimension $N \times N$, $m_{\text {sky }}, f_{\text {see }}$ and $\sigma_{\mathrm{T}}$ is shown. This is relevant since, referring to the subsequent Tables 3-5, one can verify that the results for the Reference model (in the last two rows) scale with $\left\langle u_{\mathrm{T}}\right\rangle_{\phi}$ (in Tables 3 and 4) and $\left\langle u_{\mathrm{T}}^{2}\right\rangle_{\phi}$ (last four rows in Table 5). Therefore, since $\left\langle u_{\mathrm{T}}(x, y)\right\rangle_{\phi}$ and $\left\langle u_{\mathrm{T}}^{2}(x, y)\right\rangle_{\phi}$ strongly depend on the above mentioned parameters, we expect that all pixel lensing estimated quantities heavily depend on the observing conditions and telescope capabilities.

\subsection{Pixel lensing optical depth}

Classical microlensing optical depth maps for selected M 31 source and lens populations are shown in Fig. 3 for the Reference model.

Here and below, sources and lenses in the M31 bulge and disk are indicated by indices 1 and 2, while lenses in the M 31 halo and MW disk and halo by indices 3, 5 and 6 . The first and second indices refer to source and lens, respectively. 
Table 5. The instantaneous number of events in pixel lensing observations towards the M 31 galaxy for different locations of sources and lenses is shown (for details see text). Numbers in brackets refer to the South Semisphere of M31. For the MW disk and halo, lenses in the South Semisphere of the MW contribute to roughly one half of the total and so the corresponding event numbers are not given.

\begin{tabular}{ccccccccc}
\hline \hline Model & $\left\langle u_{\mathrm{T}}^{2}\right\rangle_{\phi}$ & Bulge & Disk & M31 halo & M31 overall & Stellar & M31 halo & 23 \\
\hline Massive halo & $9.56 \times 10^{-3}$ & 0.74 & 0.57 & 2.25 & 3.56 & $0.68 / 0.63(1.08)$ & $1.52 / 0.73(2.09)$ & $1.16 / 0.42(2.74)$ \\
Massive bulge & & 2.22 & 2.87 & 1.37 & 6.45 & $1.74 / 3.34(0.52)$ & $0.83 / 0.54(1.55)$ & $0.41 / 0.17(2.46)$ \\
Massive disk & & 0.74 & 3.35 & 0.98 & 5.07 & $1.32 / 2.77(0.48)$ & $0.64 / 0.34(1.86)$ & $0.49 / 0.21(2.29)$ \\
Reference & & 0.70 & 0.90 & 1.78 & 3.37 & $0.69 / 0.90(0.77)$ & $1.21 / 0.57(2.11)$ & $0.89 / 0.31(2.89)$ \\
\hline Massive halo & \multirow{2}{*}{$1.17 \times 10^{-3}$} & 0.08 & 0.05 & 1.34 & 1.47 & $0.09 / 0.05(1.84)$ & $0.95 / 0.39(2.47)$ & $0.85 / 0.30(2.82)$ \\
Massive bulge & & 0.20 & 0.20 & 0.67 & 1.08 & $0.18 / 0.22(0.82)$ & $0.44 / 0.23(1.88)$ & $0.32 / 0.13(2.50)$ \\
Massive disk & & 0.09 & 0.29 & 0.58 & 0.96 & $0.17 / 0.21(0.81)$ & $0.39 / 0.19(2.10)$ & $0.35 / 0.15(2.32)$ \\
Reference & & 0.08 & 0.07 & 1.04 & 1.19 & $0.09 / 0.07(1.32)$ & $0.74 / 0.29(2.52)$ & $0.66 / 0.22(2.96)$ \\
\hline
\end{tabular}

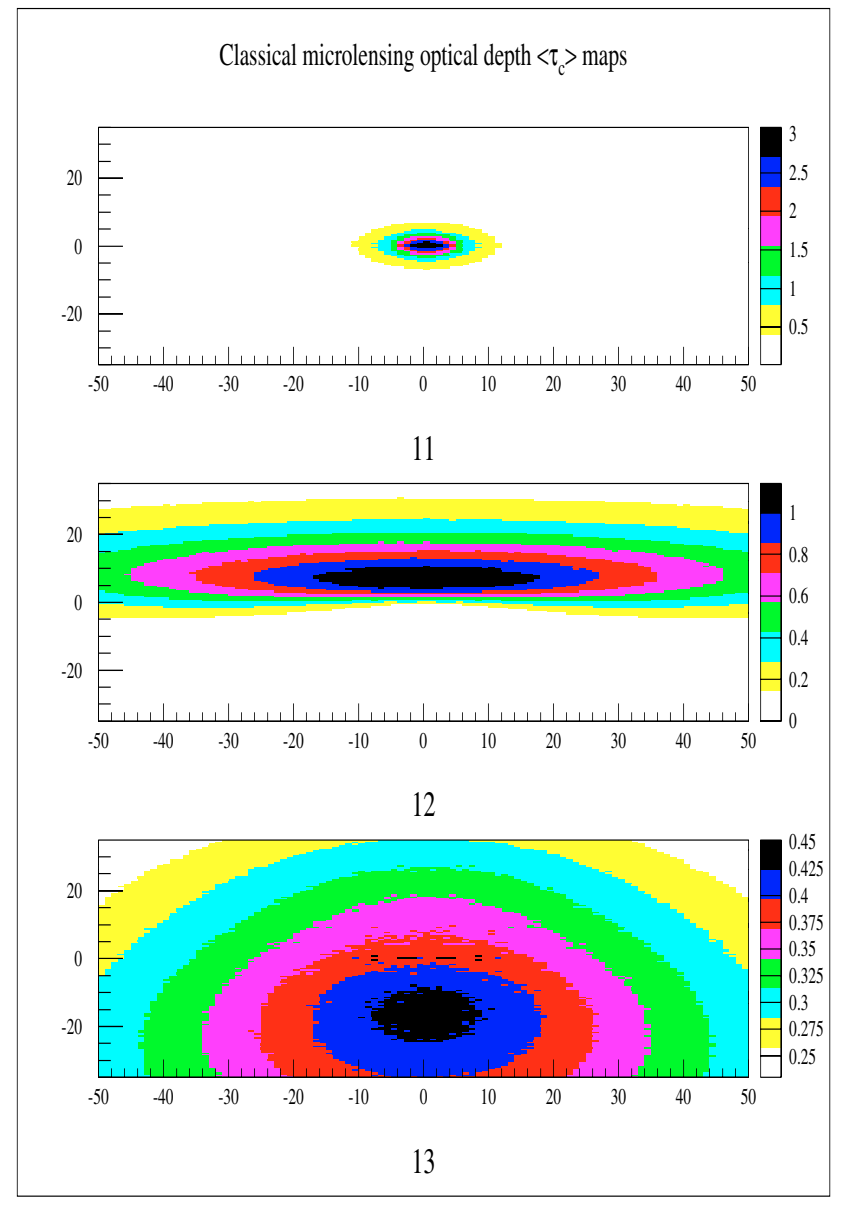

a)

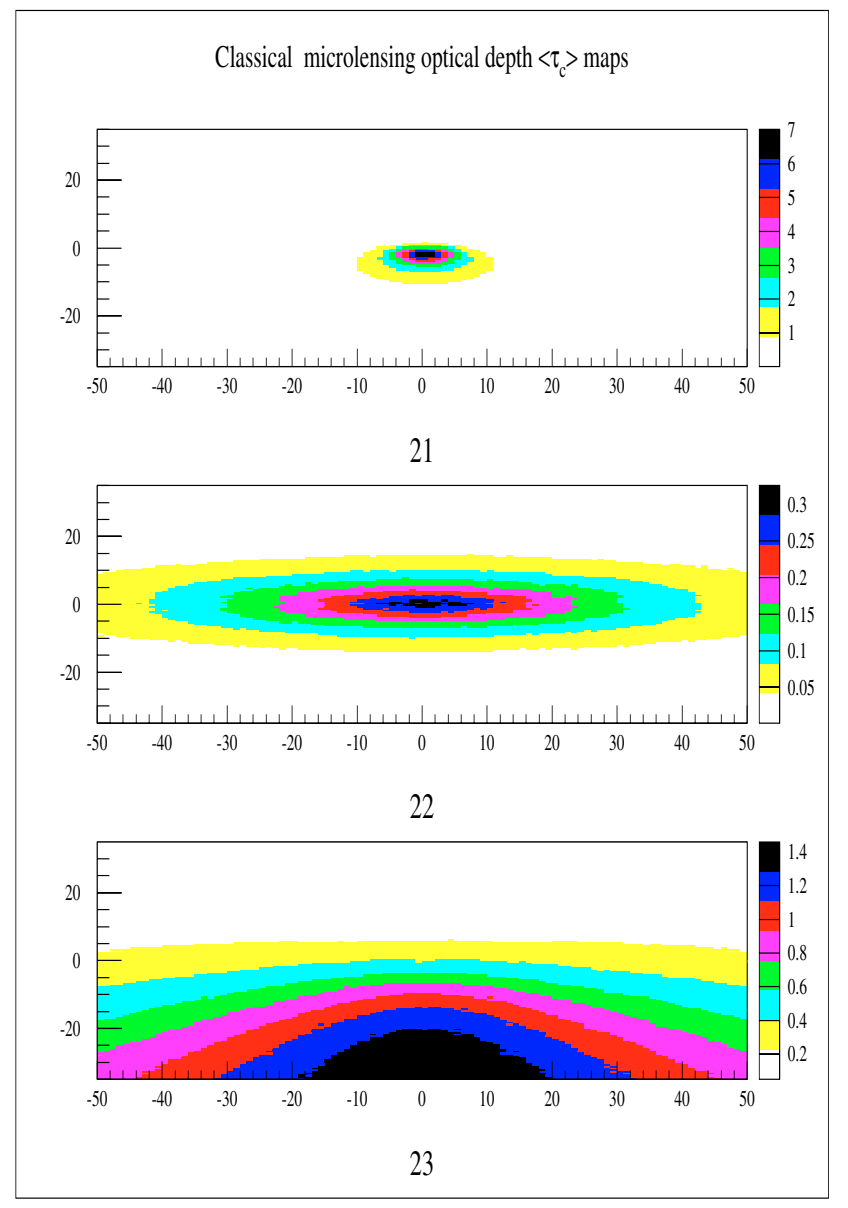

b)

Fig. 3. Mean optical depth $\left\langle\tau_{\mathrm{c}}(x, y)\right\rangle$ maps (in units of $\left.10^{-6}\right)$ towards $\mathrm{M} 31$ are given for selected source and lens populations. The first index refers to the source stars ( 1 for M 31 bulge, 2 for M 31 disk) while the second one refers to the lens populations ( 1 and 2 as above, 3 for MACHOs in the M 31 halo). Optical depth maps for lenses belonging to the MW disk and halo populations are not given since the obtained results are almost constant in any direction.

As one can see, $\left\langle\tau_{c}(x, y)\right\rangle$ always increases towards the M31 center. The well-known far-to-near side asymmetry of the M31 disk is clearly demonstrated in $\left\langle\tau_{\mathrm{c}}(x, y)\right\rangle_{23}$, where the lenses are in the M 31 halo. Moreover, a strong asymmetry in the opposite direction in the bulge-disk (12) and diskbulge (21) events (due to the relative source-lens location) is also evident.
We have also found that the classical mean optical depth $\left\langle\tau_{\mathrm{c}}(x, y)\right\rangle$ for lenses in our Galaxy $\left(\left\langle\tau_{\mathrm{c}}(x, y)\right\rangle_{15},\left\langle\tau_{\mathrm{c}}(x, y)\right\rangle_{25}\right.$, $\left\langle\tau_{\mathrm{c}}(x, y)\right\rangle_{16}$ and $\left.\left\langle\tau_{\mathrm{c}}(x, y)\right\rangle_{26}\right)$ is almost constant in any direction and therefore we do not show the corresponding maps. For reference, the obtained values are $\left\langle\tau_{\mathrm{c}}\right\rangle_{15} \simeq\left\langle\tau_{\mathrm{c}}\right\rangle_{25} \simeq 0.03 \times 10^{-6}$ and $\left\langle\tau_{\mathrm{c}}\right\rangle_{16} \simeq\left\langle\tau_{\mathrm{c}}\right\rangle_{26} \simeq 0.23 \times 10^{-6}$. 


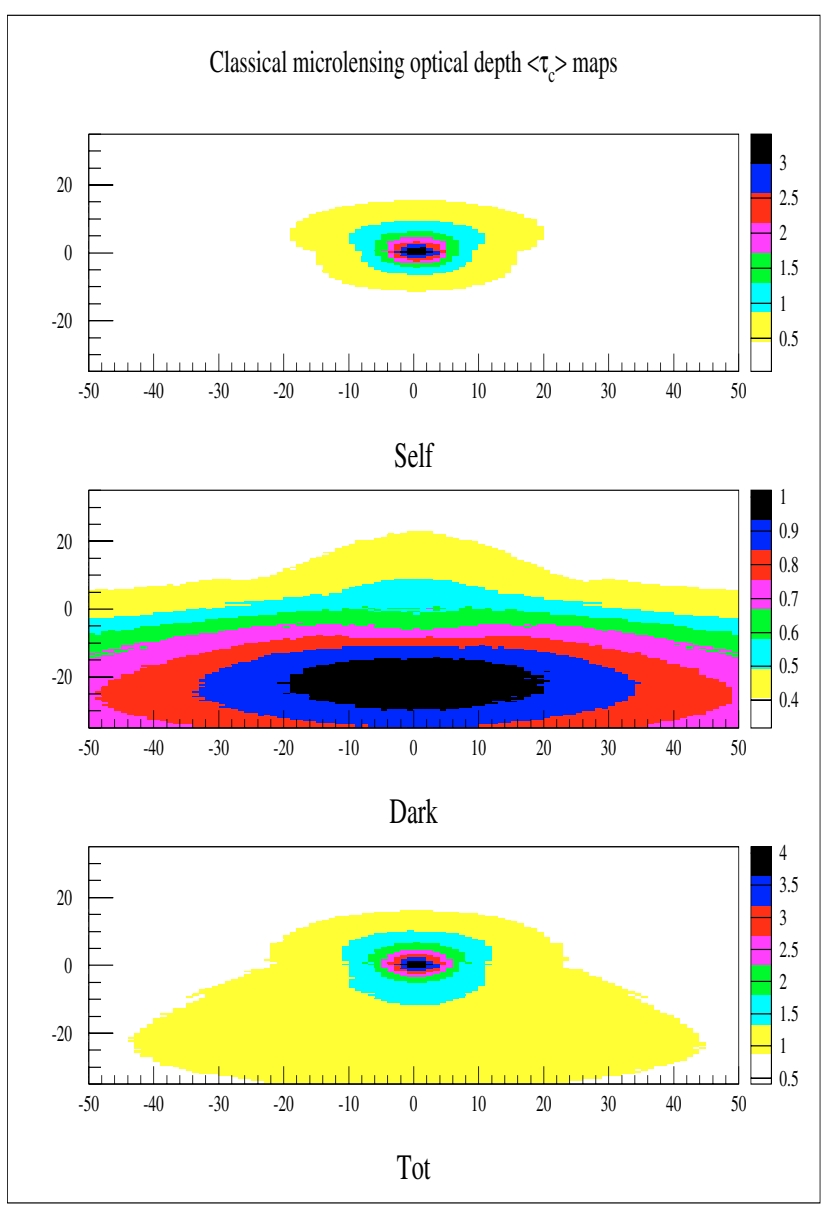

a)

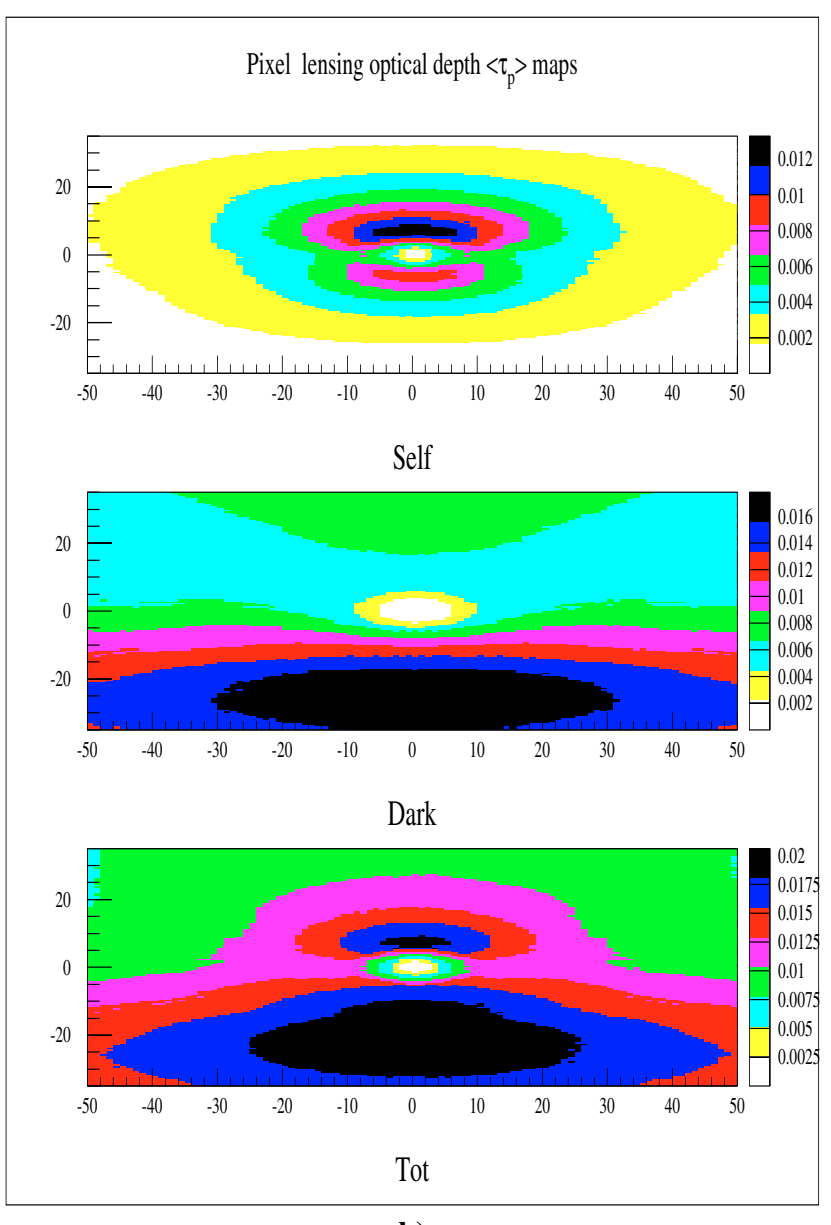

b)

Fig. 4. In panel a), the mean classical optical depth $\left\langle\tau_{c}(x, y)\right\rangle$ maps (in units of $10^{-6}$ ) towards M 31 are given for self, dark and total lensing. In panel b), the mean pixel lensing optical depth $\left\langle\tau_{\mathrm{p}}(x, y)\right\rangle$ maps are given, in the same cases.

In Fig. 4a classical optical depth maps towards M 31 are given for self-lensing $\left(\tau_{\text {self }}=\tau_{11}+\tau_{12}+\tau_{21}+\tau_{22}\right)$ and darklensing $\left(\tau_{\text {dark }}=\tau_{13}+\tau_{16}+\tau_{23}+\tau_{26}\right)$. The total contribution $\tau_{\text {tot }}=\tau_{\text {self }}+\tau_{\text {dark }}$ is given at the bottom of the same figure.

We notice that, in order to evaluate $\tau_{\text {self }}$ and $\tau_{\text {dark }}$, we sum optical depths obtained for different source populations and therefore the averaging procedure in Eq. (30) is done by normalizing with the factor $\int\left[n_{1}\left(D_{\mathrm{s}} ; x, y\right)+n_{2}\left(D_{\mathrm{s}} ; x, y\right)\right] \mathrm{d} D_{\mathrm{s}}$. Mean pixel lensing optical depth $\left\langle\tau_{\mathrm{p}}(x, y)\right\rangle$ maps are shown in Fig. $4 \mathrm{~b}$.

As we can see, the main effect of the threshold impact parameter is to substantially decrease $\left\langle\tau_{\mathrm{p}}(x, y)\right\rangle$ (with respect to $\left\langle\tau_{\mathrm{c}}(x, y)\right\rangle$ values) in particular towards the central regions of M 31, as a consequence of the increasing luminosity. Indeed, on average $\left\langle\tau_{\mathrm{p}}\right\rangle \simeq\left\langle u_{\mathrm{T}}^{2}\right\rangle_{\phi}\left\langle\tau_{\mathrm{c}}\right\rangle$ and $\left\langle u_{\mathrm{T}}^{2}\right\rangle_{\phi} \simeq 10^{-2}$ (see the third row in Table 2) for the parameter values used in the figures.

\subsection{Pixel lensing rate and expected event number}

Maps of the expected number of events in pixel lensing surveys towards M 31 are shown in Fig. 5 for the Reference model. As for the optical depth, we give the number of events separately for self-lensing, dark-lensing and also the total contribution.
In Figs. 5a and 5b we show, as a function of position, maps of the instantaneous event number density $\left\langle\operatorname{IE}_{\mathrm{p}}(x, y)\right\rangle$ (events per $\operatorname{arcmin}^{2}$ ) and the event rate $\left\langle E_{\mathrm{p}}(x, y)\right\rangle$ (events per year and $\operatorname{arcmin}^{2}$ ).

or the optical depth, the effect of the threshold impact parameter is to produce a decrease of the event number density towards the M 31 center (for $r \lesssim 2$ arcmin) and an overall reduction of the event number density with respect to the expectations from classical microlensing results. Moreover, in the figures it is also evident that the inner region (within about 10 arcmin from the M 31 center) is dominated by self-lensing events.

In Fig. 7 the projected (along the $x$ axis) mean event number density $\left\langle E_{\mathrm{p}}(y)\right\rangle$ as a function of the coordinate $y$ is given. The dashed line refers to dark lensing events by MACHOs in M 31 and MW halos while the solid line is for self-lensing events by stars in M 31 bulge and disk. The North/South asymmetry is evident for dark events that are relatively more numerous in the South Semisphere, corresponding to the far side of the M31 disk.

In Table 3, for selected locations of sources (stars in M 31 bulge and disk) and lenses (stars in M 31 bulge and disk, stars in MW disk and MACHOs in M 31 and MW halos) we give the 


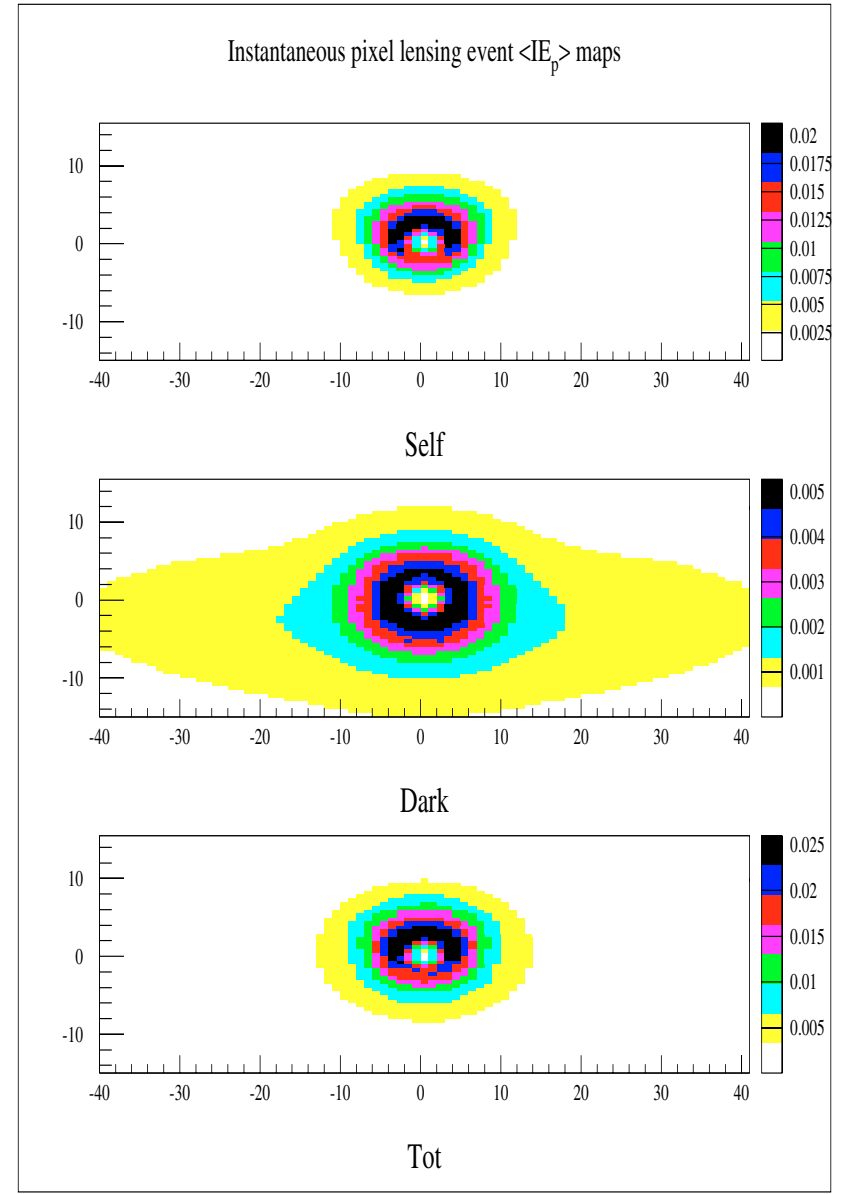

a)

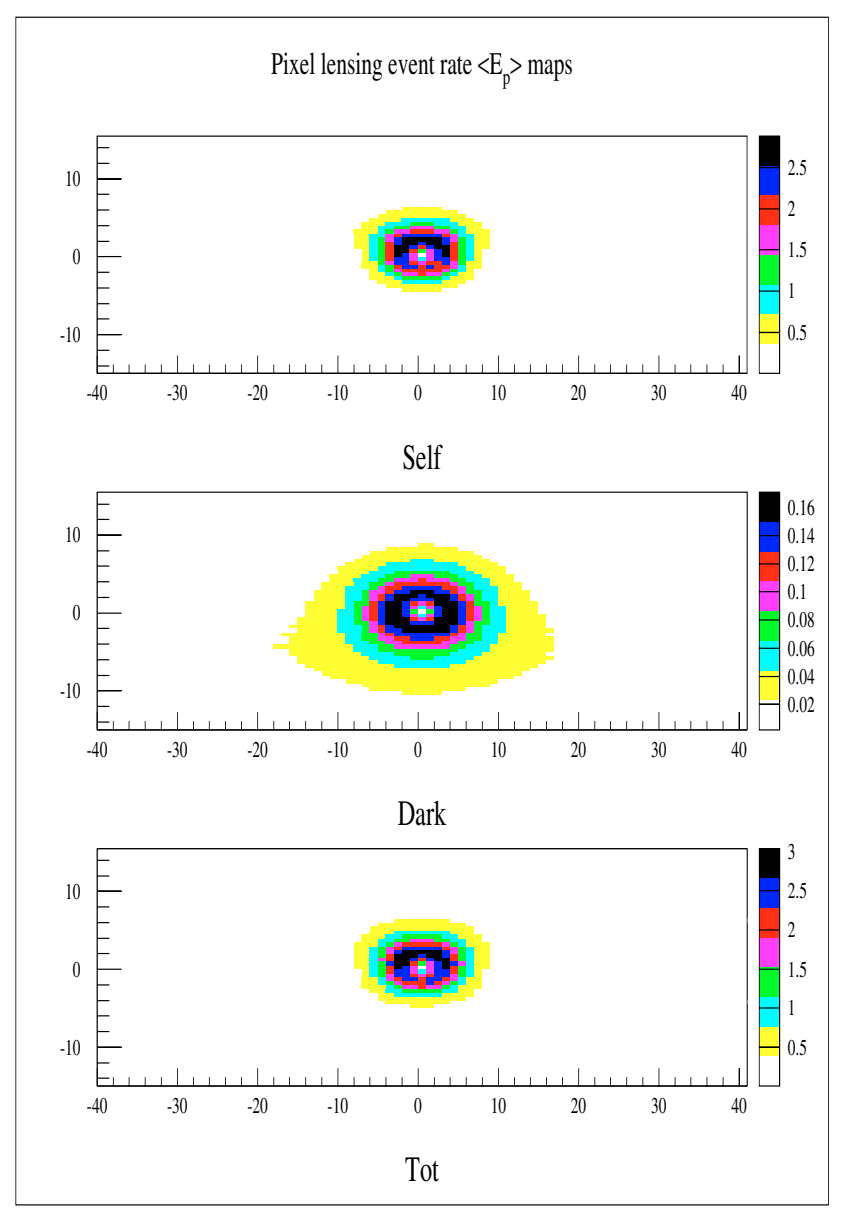

b)

Fig. 5. In panel a), the instantaneous pixel lensing event number density $\left\langle I E_{\mathrm{p}}(x, y)\right\rangle$ maps (events per $\left.\operatorname{arcmin}^{2}\right)$ towards $\mathrm{M} 31$ are given for self, dark and total lensing. In panel b) maps of pixel lensing event rate $\left\langle E_{\mathrm{p}}(x, y)\right\rangle$ (events per year and per $\left.\operatorname{arcmin}^{2}\right)$ are given, in the same cases.

expected total number of events detectable by monitoring for 1 year the $100 \times 70 \mathrm{arcmin}^{2}$ region oriented along the major axis of M 31 (events within 8 arcmin from the center are excluded). The first four lines refer to the models considered in Table 1 and to the parameters in the third row of Table 2. As one can see, the obtained results for the Reference model are intermediate with respect to those for the other more extreme models.

In the last row of Table 3, for the Reference model we show how the expected event number changes considering a different value of $\left\langle u_{\mathrm{T}}\right\rangle_{\phi} \simeq 1.44 \times 10^{-2}$ (see 5 th row in Table 2 ). As expected, one can verify that roughly the event number scales as $\left\langle u_{\mathrm{T}}\right\rangle_{\phi}$.

Similar results have been obtained in previous simulations (see, e.g. Kerins 2004, and references therein). We also note that our numerical results scale with the fraction of halo dark matter in form of MACHOs and with the MACHO mass by a factor $\left(f_{\mathrm{MACHO}} / 0.2\right) \sqrt{0.5 M_{\odot} / m_{1}}$.

In Table 4 we give the total event number $\left\langle E_{\mathrm{p}}\right\rangle$ for different lens populations (bulge, disk and halo) located in M31. As one can see, the ratio dark/total events depends on the considered model, varying from 0.07 (for the massive disk model) to 0.40 for the massive halo model.
To study the far-disk/near-disk asymmetry, in the last three columns of Table 4 we give results for the South/North M31 Semispheres and in brackets their ratio. For the Reference model, we find that self-lensing events are roughly symmetric (the same is true for lenses located in the MW disk and halo, not given in the table), while events due to lenses in M31 halo are asymmetrically distributed with a ratio of about 2 . The asymmetry is particularly evident (in the last column of the table) for sources located in the disk.

In Table 5 the instantaneous total number of events $\left\langle I E_{\mathrm{p}}\right\rangle$ within the considered M 31 region is given. The first four rows refer to the parameter values $\left\langle m_{\mathrm{b}}\right\rangle \simeq 0.31 M_{\odot},\left\langle m_{\mathrm{d}}\right\rangle \simeq 0.53 M_{\odot}$, $f_{\mathrm{MACHO}}=0.2$ and $\left\langle u_{\mathrm{T}}{ }^{2}\right\rangle_{\phi} \simeq 9.56 \times 10^{-3}$ (used throughout the paper). For comparison with the results obtained by Kerins (2004), in the last four rows of Table 5 we present our results for $\left\langle m_{\mathrm{b}}\right\rangle \simeq 0.5 M_{\odot},\left\langle m_{\mathrm{d}}\right\rangle \simeq 0.5 M_{\odot}, f_{\mathrm{MACHO}}=1$ and $\left\langle u_{\mathrm{T}}^{2}\right\rangle_{\phi} \simeq 1.17 \times 10^{-3}$. The asymmetry ratio we obtain is always rather smaller than that quoted by Kerins (2004).

As it has been mentioned by several authors, in order to discriminate between self and dark lensing events, it is important to analyze the event duration. Indeed self-lensing events are expected to have, on average, shorter duration with respect to events due to halo MACHOs. 


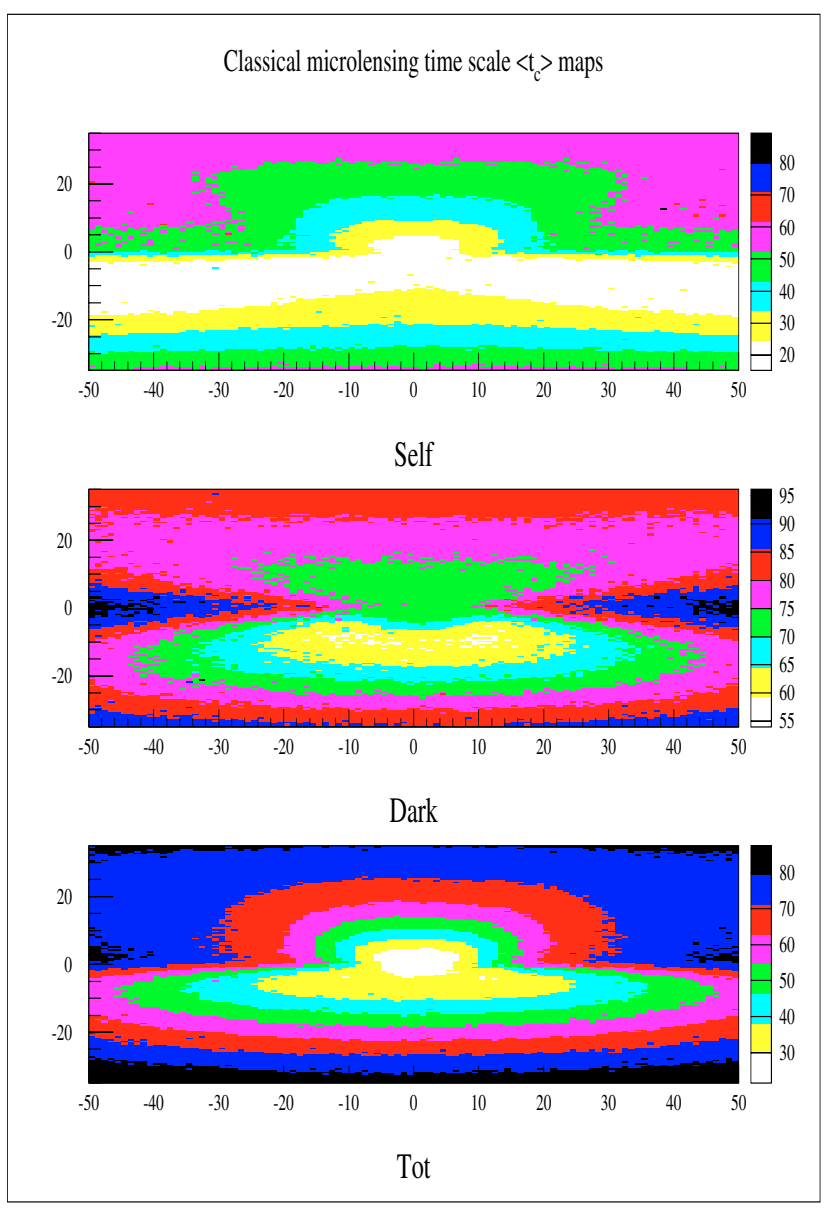

a)

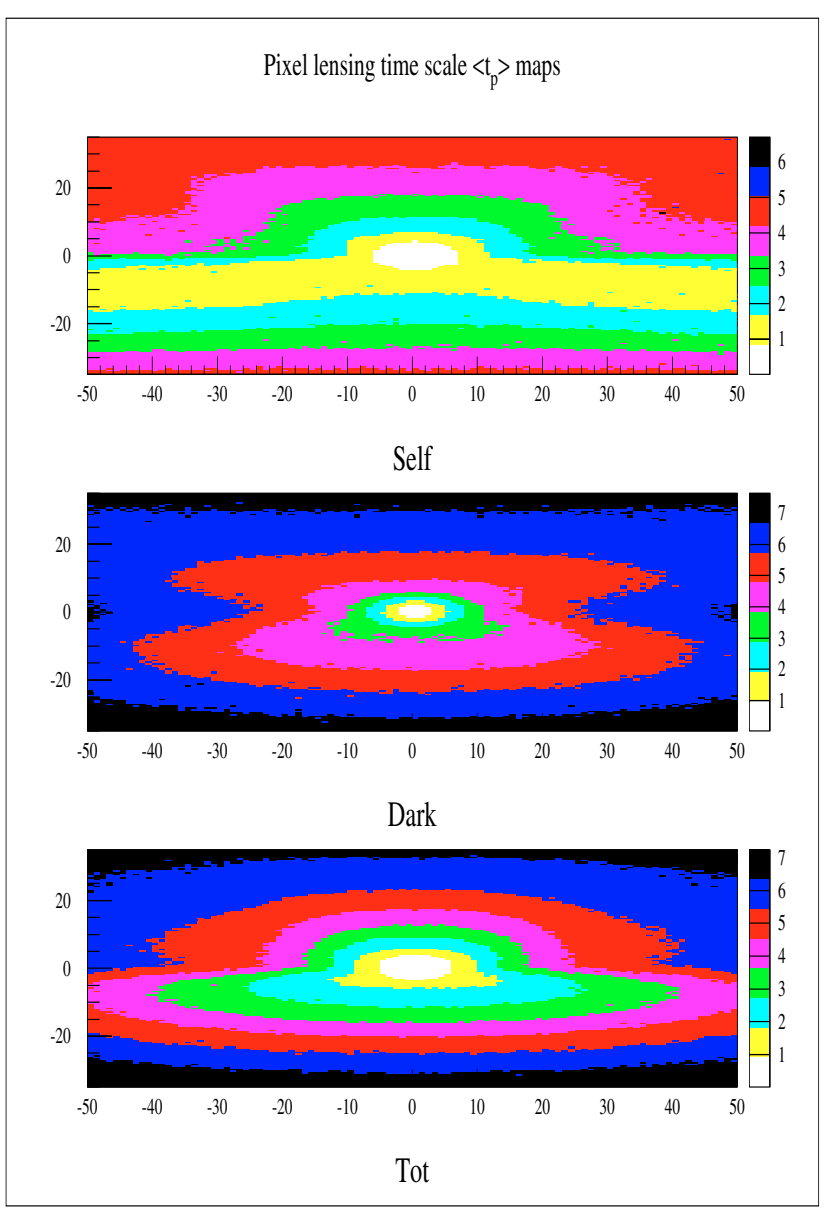

b)

Fig. 6. In panel a), mean classical event duration time $\left\langle t_{\mathrm{c}}(x, y)\right\rangle$ (in days) maps towards M31 are given for self, dark and total lensing. In panel b) for pixel lensing, maps of $\left\langle t_{\mathrm{p}}(x, y)\right\rangle$ are given, in the same cases.

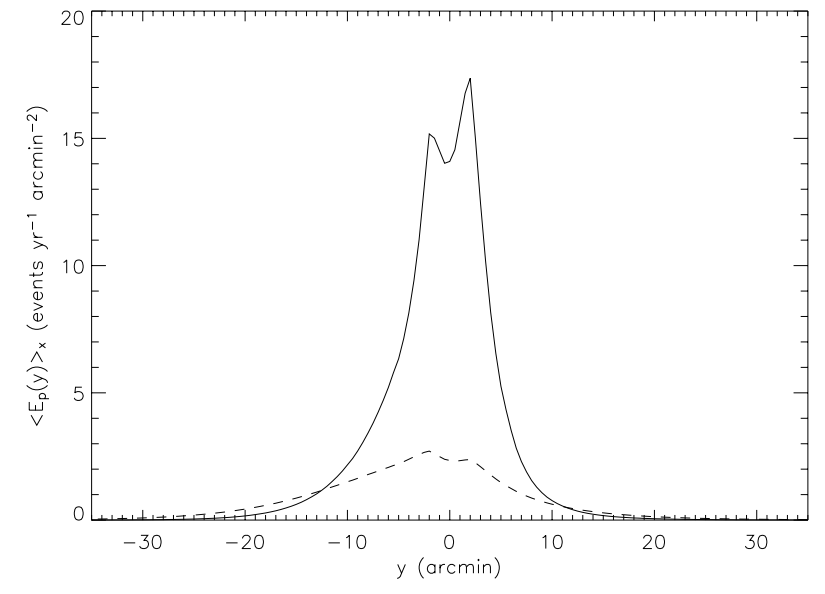

Fig. 7. The projected (along the $x$ axis) mean event number $\left\langle E_{\mathrm{p}}(y)\right\rangle_{x}$ is given as a function of the coordinate $y$ for the Reference model. The dashed line refers to dark lensing events by MACHOs in M31 and MW halos while the solid line is for self-lensing events by stars in M 31 bulge and disk.

\subsection{Pixel lensing event time scale}

Maps of mean event duration time scale in classical and pixel lensing are shown in Figs. 6a and 6b.
Here we use the probability, for each location of sources and lenses given in Eq. (26), of obtaining event duration maps for self and dark microlensing events.

As expected, short duration events are mainly distributed towards the inner regions of the galaxy and this occurs for both $\left\langle t_{\mathrm{c}}(x, y)\right\rangle$ and $\left\langle t_{\mathrm{p}}(x, y)\right\rangle$. The main effect of $\left\langle u_{\mathrm{T}}(x, y)\right\rangle_{\phi}$ is to decrease the event time scale, in particular towards the inner regions of M 31, giving a larger number of short duration events with respect to expectations based on $\left\langle t_{\mathrm{c}}(x, y)\right\rangle$ calculations.

Both for self and dark events the pixel lensing time scale we obtain is $\simeq 1-7$ days, in agreement with results in Kerins (2004), but much shorter with respect to the duration of the events observed by the MEGA Collaboration (de Jong et al. 2004). This is most likely due to the fact that current experiments may not detect events shorter than a few days.

However, the pixel lensing time scale values depend on $\left\langle u_{\mathrm{T}}(x, y)\right\rangle_{\phi}$ and ultimately on the observational conditions and the adopted analysis procedure. Indeed from Table 2 one can see that the $\left\langle u_{\mathrm{T}}(x, y)\right\rangle_{\phi}$ value may be easily doubled, changing the adopted parameters and therefore giving longer events.

In Fig. 8 the pixel lensing event duration $\left\langle t_{\mathrm{p}}(y)\right\rangle$ averaged along the $x$ direction is given as a function of the $y$ coordinate. The dashed line refers to dark lensing events by MACHOs 


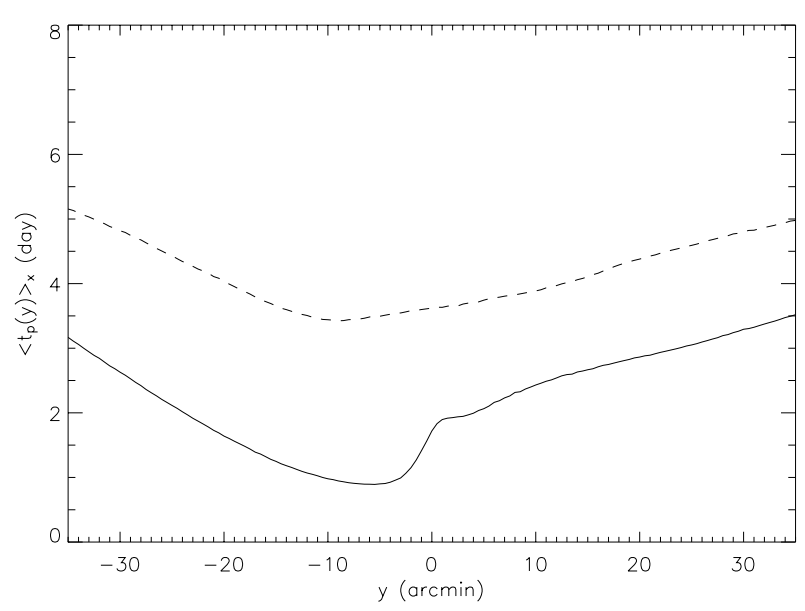

Fig. 8. The pixel lensing event duration $\left\langle t_{\mathrm{p}}(y)\right\rangle_{x}$ averaged along the $x$ direction is given as a function of the $y$ coordinate. The dashed line refers to dark lensing events by MACHOs in M 31 and MW halos while the solid line is for self-lensing events by stars in M 31 bulge and disk.

in M31 and MW halos while the solid line is for self-lensing events by stars in M 31 bulge and disk.

It is clearly evident that dark events last roughly twice as long as self-lensing events and that the shortest events are expected to occur towards the M31 South Semisphere.

The presence of a large number of short duration events in pixel lensing experiments towards M31 has been reported by several authors (Paulin-Henriksson et al. 2003; Paulin-Henriksson 2004).

\section{Conclusions}

We have studied the optical depth, event number and time scale distributions in pixel lensing surveys towards M 31 by addressing, in particular, the changes with respect to expectations from classical microlensing (in which the sources are resolved).

Assuming, as reference values, the capabilities of the Isaac Newton Telescope in La Palma and typical CCD camera parameters, exposure time and background photon counts, we perform an analysis consisting of averaging all relevant microlensing quantities over the threshold value $u_{\mathrm{T}}(x, y)$ of the impact parameter. Clearly, as in classical microlensing estimates, an average procedure is also done with respect to all the other parameters entering in microlensing observables: source and lens position, lens mass and source and lens transverse velocities.

The M 31 bulge, disk and halo mass distributions are described following the Reference model in Kerins (2004), which provides remarkably good fits to the M31 surface brightness and rotation curve profiles. We also take a standard mass distribution model for the MW galaxy, as described in Sect. 2, and assume that M31 and MW halos contain 20\% $0.5 M_{\odot}$ MACHOs.

We consider red giants as the sources that most likely may be magnified (and detected in the red band) in microlensing surveys. Moreover, given the lack of precise information about the stellar luminosity function in M31, we assume that the same function holds both for the Galaxy and M31 and does not depend on the position. Accordingly, the fraction of red giants (over the total star number) is $\simeq 5.3 \times 10^{-3}$.

Our main results are maps in the sky plane towards M 31 of threshold impact parameter $\left\langle u_{\mathrm{T}}(x, y)\right\rangle_{\phi}$, optical depth $\left\langle\tau_{\mathrm{p}}(x, y)\right\rangle$, instantaneous event number density $\left\langle I E_{\mathrm{p}}(x, y)\right\rangle$ (events per $\operatorname{arcmin}^{2}$ of ongoing microlensing events at any instant in time) and event number density $\left\langle E_{\mathrm{p}}(x, y)\right\rangle$ (events per yr and $\operatorname{arcmin}^{2}$ to be detected in M 31 surveys) and time scale $\left\langle t_{\mathrm{p}}(x, y)\right\rangle$.

These maps show an overall reduction of the corresponding classical microlensing results and also a distortion of their shapes with respect to other results in the literature.

Figures 3 and 4 show maps of the mean optical depth (averaged over the source number density) for the different source and lens locations.

In Fig. 5 we give the instantaneous pixel lensing event number density and the event rate for self, dark and total lensing. It clearly appears that the central region of M 31 is dominated by self-lensing events due to sources and lenses in M31 itself, while dark events are relatively more numerous in the outer region (see also Fig. 7).

In Tables 3 and 4, for the M31 mass distribution models considered by Kerins (2004), we give the expected total event number $\left\langle E_{\mathrm{p}}\right\rangle$ to be detected by monitoring, for $1 \mathrm{yr}$, the $100 \times 70 \mathrm{arcmin}^{2}$ region oriented along the major axis of M31 (the inner 8 arcmin region is excluded). We find that the expected dark to total event number ratio is between $7 \%$ (for the massive disk model) and $40 \%$ (for the massive halo model). The tables also show the well-known far-disk/neardisk asymmetry due to lenses in the M31 halo. Self-lensing events, instead, are distributed more symmetrically between the M31 North and South Semisphere. Similar conclusions are evident from Table 5, where we give the instantaneous number of events, although the asymmetry ratio we obtain is always smaller than the values quoted by Kerins (2004).

Figure 6 shows a decrease of the event time scale with respect to classical microlensing, particularly towards the inner regions of M 31, due to the high brightness of the galaxy. Both for self and dark lensing events, the pixel lensing time scale we obtain is $\simeq 1-7$ days, in agreement with results in the literature. Note that the duration of the events observed by the MEGA Collaboration (de Jong et al. 2004) is typically much longer than 7 days, due to the difficulty of detecting short events in current experiments. It is also clear from Fig. 6 that dark events last roughly twice as long as self-lensing events and that the shortest events are expected to occur towards the M31 South Semisphere (see Fig. 8).

However, we emphasize that the pixel lensing results obtained depend on $\left\langle u_{\mathrm{T}}(x, y)\right\rangle_{\phi}$ and $\left\langle u_{\mathrm{T}}^{2}(x, y)\right\rangle_{\phi}$ values, and ultimately on the observing conditions and telescope capabilities. Indeed, from Table 2, where the values of $\left\langle u_{\mathrm{T}}\right\rangle_{\phi}$ and $\left\langle u_{\mathrm{T}}^{2}\right\rangle_{\phi}$ averaged over the whole M31 galaxy are given, one can verify that pixel lensing quantities scaling with $\left\langle u_{\mathrm{T}}\right\rangle_{\phi}\left(\left\langle E_{\mathrm{p}}\right\rangle\right.$ and $\left.\left\langle t_{\mathrm{p}}\right\rangle\right)$ may vary by more than one order of magnitude while quantities scaling with $\left\langle u_{\mathrm{T}}^{2}\right\rangle_{\phi}\left(\left\langle\tau_{\mathrm{p}}\right\rangle\right.$ and $\left.\left\langle I E_{\mathrm{p}}\right\rangle\right)$ may change by two orders of magnitude.

The present analysis can be used to test estimates and Monte-Carlo simulations by other Collaborations and it has 
also been performed in view of a planned survey towards M 31 by the SLOTT-AGAPE Collaboration (Bozza et al. 2000).

Acknowledgements. We acknowledge S. Calchi Novati, Ph. Jetzer and F. Strafella for useful discussions.

\section{References}

Alcock, C., Akerloff, C. W., Allsman, R. A., et al. 1993, Nature, 365, 621

Alcock, C., Allsman, R. A., Alves, D. R., et al. 2000, ApJ, 542, 281

Ansari, R., Auriere, M., Baillon, P., et al. 1995, in Proc. of the XVII Texas Symp., Annals of the N.Y. Academy of Sciences, ed. Bohringer H., Morfill G. E., \& Trumper J. E., 759, 608

Ansari, R., Auriere, M., Baillon, P., et al. 1997, A\&A, 324, 843

Ansari, R., Auriere, M., Baillon, P., et al. 1999, A\&A, 344, L49

An, J. H., Evans, N. W., Kerins, E., et al. 2004, ApJ, 601, 845

Aubourg, E., Bareyre, P., Brehin, S., et al. 1993, Nature, 365, 623

Auriere, M., Baillon, P., Bouquet, A., et al. 2001, ApJ, 553, L137

Baillon, P., Bouquet, A., Giraud-Heraud, Y., \& Kaplan, J. 1993, A\&A, 277,1

Baltz, E. A., Gyuk, G., \& Crotts, A. P. S. 2003, ApJ, 582, 30

Bozza, V., Calchi Novati, S., Capaccioli, M., et al. 2000, Mem. Soc. Astron. It., 71, 1113

Calchi Novati, S., Iovane, G., Marino, A., et al. 2002, A\&A, 381, 845

Calchi Novati, S., Jetzer, Ph., Scarpetta, G., et al. 2003, A\&A, 405, 851

Caon, N., Capaccioli, M., \& D’Onofrio, M. 1993, MNRAS 265, 1013
Colley, W. N. 1995, AJ, 109, 440

Crotts, A. P. S. 1992, ApJ, 399, L43

Crotts, A. P. S., \& Tomaney, A. B. 1996, ApJ, 473, L87

de Jong, J. T. A., Kuijken, K., Crotts, A. P. S., et al. 2004, A\&A, 417, 461

De Paolis, F., Ingrosso, G., Nucita, A., et al. 2004, in preparation

Dwek, E., Arendt, R. G., Hauser, M. G., et al. 1995, ApJ, 445, 716

Giudice, G. F., Mollerach, S., \& Roulet, E. 1994, Phys. Rev. D, 50, 4 Gondolo, P. 1999, ApJ, 510, L29

Gould, A. 1994, ApJ, 435, 573

Griest, K. 1991, ApJ, 366, 412

Gyuk, G., \& Crotts, A. P. S. 2000, ApJ, 535, 621

Han, C., \& Gould, A. 1996, ApJ, 473, 230

Jetzer, Ph. 1994, A\&A, 286, 426

Kent, S. M. 1989, AJ, 97, 1614

Kerins, E., Carr, B., Ewans, N.W., et al. 2001, MNRAS, 323, 13

Kerins, E., An, J., Ewans, N. W., et al. 2003, ApJ, 598, 993

Kerins, E. 2004, MNRAS, 347, 1033

Mamon, G. A., \& Soneira, R. M. 1982, ApJ, 255, 181

Paczynski, B. 1986, ApJ, 304, 1

Paulin-Henriksson S., Baillon P., Bouquet A., et al. 2003, A\&A, 405, 15

Paulin-Henriksson, S. 2004, talk at Moriond Conference, http://moriond.in2p3.fr

Reitzel, D., Guhathakurta, P., \& Gould, A. 1998, AJ, 116, 707

Riffeser, A., Fliri, J., Bender, R., et al. 2003, ApJ, 599, L17

Uglesich, R. R., Crotts, A. P. S., \& Baltz, E. A. 2004

[arXiv: astro-ph/0403248] 